\title{
Erratum
}

\section{Affinity of antineoplastic amino acid drugs for the large neutral amino acid transporter of the blood-brain barrier}

\author{
Yoshiaki Takada1, Nigel H. Greig1, David T. Vistica ${ }^{2}$, Stanley I. Rapoport ${ }^{1}$, and Quentin R. Smith ${ }^{1}$ \\ ${ }^{1}$ Laboratory of Neurosciences, National Institute on Aging, National Institutes of Health, Bethesda, MD 20 892, USA \\ ${ }^{2}$ Laboratory of Drug Discovery, Research and Development, National Cancer Institute, Frederick Cancer Research Center, P. O. Box B. Frederick, \\ MD 21 702, USA \\ Cancer Chemother Pharmacol 29: 89-94
}

Due to an unfortunate printing error Eq. 5 on page 91 contained two symbols which should not have appeared.

Eq. 5 should have read as follows:

$\%$ Inhibition $=100\left(P A_{0}-P A_{\mathrm{i}}\right) / P A_{0} \approx 100 \mathrm{c}_{\mathrm{i}} /\left(c_{\mathrm{i}}+K_{\mathrm{i}}\right)$ 